\title{
Real-time control of electronic motion: Application to Nal
}

\section{Grønager, Michael; Henriksen, Niels Engholm}

Published in:

Journal of Chemical Physics

Link to article, DOI:

10.1063/1.477036

Publication date:

1998

Document Version

Publisher's PDF, also known as Version of record

Link back to DTU Orbit

Citation (APA):

Grønager, M., \& Henriksen, N. E. (1998). Real-time control of electronic motion: Application to Nal. Journal of Chemical Physics, 109(11), 4335-4341. https://doi.org/10.1063/1.477036

\section{General rights}

Copyright and moral rights for the publications made accessible in the public portal are retained by the authors and/or other copyright owners and it is a condition of accessing publications that users recognise and abide by the legal requirements associated with these rights.

- Users may download and print one copy of any publication from the public portal for the purpose of private study or research.

- You may not further distribute the material or use it for any profit-making activity or commercial gain

- You may freely distribute the URL identifying the publication in the public portal

If you believe that this document breaches copyright please contact us providing details, and we will remove access to the work immediately and investigate your claim. 


\title{
Real-time control of electronic motion: Application to Nal
}

\author{
Michael Grønager and Niels E. Henriksen ${ }^{\text {a) }}$ \\ Department of Chemistry, Technical University of Denmark, DTU-207, DK2800 Lyngby, Denmark
}

(Received 23 April 1998; accepted 10 June 1998)

\begin{abstract}
We study theoretically the electronic and nuclear dynamics in NaI. After a femtosecond pulse has prepared a wave packet in the first excited state, we consider the adiabatic and the nonadiabatic electronic dynamics and demonstrate explicitly that a nonstationary electron is created in $\mathrm{NaI}$ corresponding to electron transfer between $\mathrm{Na}$ and I. The electronic motion is introduced via nuclear motion, more specifically, through nonadiabatic curve crossing and the electronic motion is here on the same time scale as the nuclear motion. We show that the branching ratio between the channels $\mathrm{Na}+\mathrm{I}$ and $\mathrm{Na}^{+}+\mathrm{I}^{-}$depends on the electron distribution (i.e., where the electron "sits") prior to the time where the bond is broken by a subpicosecond half-cycle unipolar electromagnetic pulse. Thus we control, in real time, which nucleus one of the valence electrons will follow after the bond is broken. (C) 1998 American Institute of Physics. [S0021-9606(98)01335-X]
\end{abstract}

\section{INTRODUCTION}

The real-time monitoring and control of chemical reaction dynamics is at the heart of femtochemistry. ${ }^{1-3}$ One of the challenging objectives is the control of electronic motion in molecules by means of external laser fields. Real-time control of electronic motion has been suggested for atoms. The wave packet motion of Rydberg states in the hydrogen atom has, for example, been generated and controlled, ${ }^{4}$ and one can envision similar control of Rydberg electrons in molecules. From a more chemical point of view an interesting objective is the control of electron transfer during bond breaking, that is, to control which nuclei the (valence) electrons will follow when a specific bond is broken in a unimolecular reaction.

We have recently discussed such a scheme ${ }^{5,6}$ in the form of an explicitly time-dependent two-pulse laser control scheme. The basic principle we use is in the spirit of the Tannor and Rice scheme, ${ }^{7,8}$ that is, vibrational or electronic wave packets are created and controlled by time-delayed ultrashort laser pulses.

The first step in our scheme is to create a nonstationary electron corresponding to (partial) electron transfer between different atoms in a molecule., ${ }^{5,6}$ The electronic motion is introduced via nuclear motion through a nonadiabatic curve crossing. We focus on electronic motion corresponding to charge transfer, that is the curve crossing is associated with electronic states which asymptotically correspond to ionic and covalent products, respectively. In an adiabatic representation, the molecular wave function has components in more than one electronic state when the wave packet moves through the crossing region and the electrons are, accordingly, nonstationary. The electron density oscillates on the time scale of nuclear motion when the wave packet oscillates back and forth through the coupling region. The second step in the scheme is to break the chemical bond between the atoms at an appropriate time. Thus depending on the electron

${ }^{\text {a)} E l e c t r o n i c ~ m a i l: ~ n e h @ t k e m i . k l b . d t u . d k ~}$ distribution prior to the time where the bond is broken by a femtosecond pulse, we can (at least, partially) control electron transfer between the separated atoms.

We have recently applied the scheme to $\mathrm{HD}^{+}$(Ref. 6) where the aim was to control the branching ratio between the channels $\mathrm{H}+\mathrm{D}^{+}$and $\mathrm{H}^{+}+\mathrm{D}$. For $\mathrm{HD}^{+}$the complete electronic and nuclear dynamics can be determined exactly within the nonrelativistic limit. The bound eigenstates of the molecule are well represented within the space of the two lowest adiabatic electronic eigenstates and we showed that a nonstationary electron density can be created by a superposition of eigenstates close to the dissociation limit. These eigenstates are highly delocalized and extend beyond an avoided crossing in the "true" adiabatic representation of the electronic states. ${ }^{6}$ The oscillating wave packet is never completely clear of the avoided crossing. Nevertheless, in the vibrating molecule partial electron transfer between the proton and the deuteron was observed and, in addition, we observed a branching ratio between the two channels which depends on the time when the bond is broken.

In this paper we consider laser control of electron transfer in NaI. The ionic ground state (asymptotically, $\mathrm{Na}^{+}$ $+\mathrm{I}^{-}$) and the lowest excited covalent state (asymptotically, $\mathrm{Na}+\mathrm{I})$ are considered, see Fig. 1. In the adiabatic representation an avoided crossing exists between these two states at an internuclear distance of about $7 \AA$. It is well-known that a femtosecond pulse can prepare a wave packet on the repulsive part of the first excited state and that this wave packet will oscillate in the adiabatic well with a fraction leaking out to form $\mathrm{Na}+$ I with each passage of the crossing. ${ }^{9,10}$

We focus on the combined electronic and nuclear dynamics and follow explicitly the adiabatic and nonadiabatic electron dynamics (where an electron "jumps" from Na to I). The present calculations on $\mathrm{NaI}$ present some new aspects compared to our study on $\mathrm{HD}^{+}$, and it is much simpler to create a nonstationary electron in $\mathrm{NaI}$ due to the presence of a deep adiabatic well. ${ }^{5}$ It is indeed well-known, at least implicitly, that charge transfer can take place during the vibration of $\mathrm{NaI}$ after it is transfered to the first excited state. The 


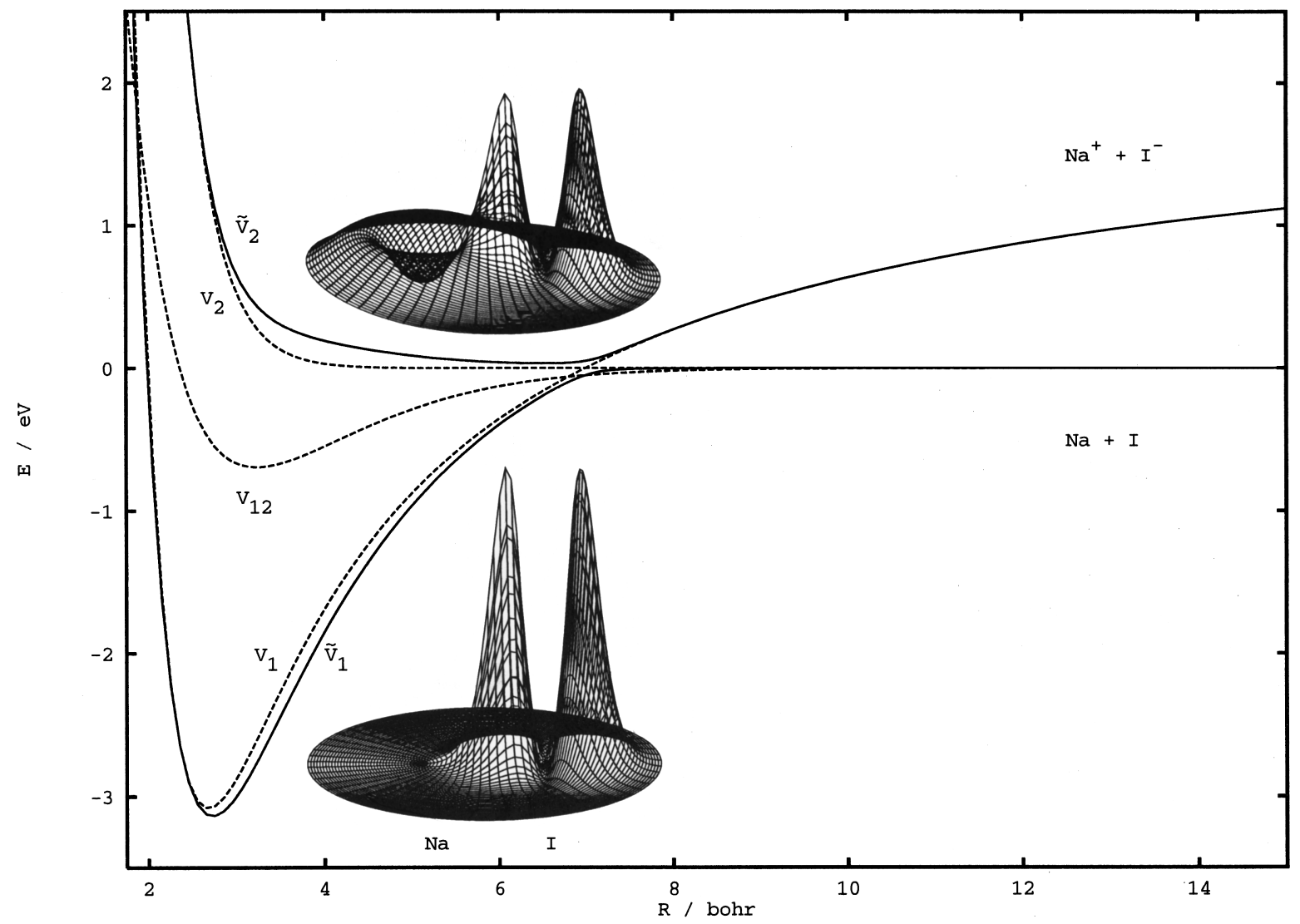

FIG. 1. The adiabatic potentials, $\widetilde{V}_{1}$ and $\widetilde{V}_{2}$ (solid) and the diabatic potentials, $V_{1}$ and $V_{2}$, and coupling, $V_{12}$ (dashed). Further, we see the electron densities (in prolate spheroidal coordinates) for each electronic state at the equilibrium distance of $R_{0}=2.7 \AA$.

basic idea is simply to take advantage of this charge transfer when the wave packet is passing the avoided crossing, that is we control the electron transfer by breaking the chemical bond before the electron distribution returns to the covalent form.

The control scheme has some similarities with an experiment carried out by Zewail and co-workers ${ }^{11}$ where the evolving dynamics in NaI was controlled. The wave packet was intercepted by a femtosecond control pulse before the crossing was reached. Thus, some fraction of the dissociative system is transfered to another potential energy surface and it was observed that population is removed from the $\mathrm{Na}+\mathrm{I}$ channel. In the present study we redirect the bond breaking in $\mathrm{NaI}$ into the ionic channel where electron transfer has taken place.

When applied to NaI the scheme consists of: (1) the preparation of an oscillating electron associated with the vibrating predissociating state in the adiabatic well [denoted by $\left.(\mathrm{NaI})^{*}\right]$, and (2) the dissociation of this nonstationary state. Thus,

$$
\stackrel{\text { Pump }}{\mathrm{NaI}} \rightarrow(\mathrm{NaI})^{*} \stackrel{\text { Control }}{\rightarrow}\left\{\begin{array}{l}
\mathrm{Na}+\mathrm{I} \\
\mathrm{Na}^{+}+\mathrm{I}^{-}
\end{array}\right.
$$

The control pulse is fired when both valence electrons "sit" on I and the reaction is then redirected from the covalent to the ionic channel. Thus the oscillating electron distribution is "caught" and "frozen" by the laser pulse that breaks the bond.

The dynamics of the NaI molecule has been studied extensively, experimentally ${ }^{9-13}$ as well as theoretically. ${ }^{14-20}$ The potential energy surfaces, the nonadiabatic coupling and the dipole moments are known. ${ }^{17,21-24}$ It is, accordingly, a well studied system where the calculations could lead the way for new experiments. These facts make NaI a good system for the control scheme described above.

The paper is organized as follows. In Sec. II, we present the semiempirical valence-bond (VB) potentials and the electronic wave functions. In Sec. III, we consider the control scheme. First we prepare and monitor $\mathrm{NaI}$ in the excited state with an oscillating electron, then we show that the use of a "half-cycle" unipolar picosecond pulse, fired at the right time has a controlling influence on the branching ratio. Section IV is the discussion and concluding remarks.

\section{VB-POTENTIALS AND ELECTRONIC WAVE FUNCTIONS}

We use the VB-potentials and electronic wave functions derived by Peslherbe et al. (see Ref. 17). We present here an outline of their results and make the connection to the electron distribution.

In semiempirical VB-theory, ${ }^{23}$ some or all of the valence 
electrons are treated explicitly, while the other electrons are embedded in a core-core potential, which is treated as an adjustable parameter. In NaI we only need to consider two valence electrons explicitly and are left with a $3 s$ orbital on $\mathrm{Na}, \varphi_{3 s}$, and a $5 p_{z}$ orbital on I, $\varphi_{5 p}$. The diabatic electronic wave functions are described by the Slater determinants $\Phi_{1}$ $=\left|\overline{\varphi_{5 p}} \varphi_{5 p}\right|$ and $\Phi_{2}=N_{2}\left(\left|\overline{\varphi_{3 s}} \varphi_{5 p}\right|-\left|\varphi_{3 s} \overline{\varphi_{5 p}}\right|\right)$ for the ionic and covalent states, respectively. $N_{2}$ is introduced due to the nonorthogonality of the $3 s$ orbital on $\mathrm{Na}$ and the $5 p_{z}$ orbital on I. The diabatic potentials, the coupling and the electronic dipole moments can now be obtained from the overlap integral $^{25}$ and the core-core potential. The adiabatic potentials and the corresponding coupling and electronic dipole moments are obtained through diagonalization of the potential energy matrix. ${ }^{26}$

The electron density, $\rho(\mathbf{r})$, can be obtained as the one electron density times the number of electrons. Hence, integrating over spin, $\sigma$, internuclear separation variable, $R$, and the coordinates of the other electron, $\mathbf{r}^{\prime}$, we get:

$$
\begin{aligned}
\rho(\mathbf{r}, t)= & 2 \int_{0}^{\infty} d R \int d \mathbf{r}^{\prime} \int d \sigma^{\prime} \int d \sigma \mid \Phi_{1}\left(\mathbf{r}, \mathbf{r}^{\prime}, \sigma, \sigma^{\prime} ; R\right) \\
& \times \chi_{1}(R, t)+\left.\Phi_{2}\left(\mathbf{r}, \mathbf{r}^{\prime}, \sigma, \sigma^{\prime} ; R\right) \chi_{2}(R, t)\right|^{2},
\end{aligned}
$$

where $\chi_{1}(R, t)$ and $\chi_{2}(R, t)$ are the nuclear wave functions residing on the lower and upper electronic potentials, respectively. The entire molecular wave function is hence formed as the sum over products of electronic, $\Phi_{n}\left(\mathbf{r}, \mathbf{r}^{\prime}, \sigma, \sigma^{\prime} ; R\right)$, and nuclear, $\chi_{n}(R, t)$, wave functions. The integrals over $\mathbf{r}^{\prime}$ and spin $\left(\sigma, \sigma^{\prime}\right)$ are easily performed. Further, it is here convenient to transform to prolate spheroidal coordinates, ${ }^{6,27}$ $(u, v, \phi)$. Due to the rotational symmetry of the bond the angular variable $\phi$ separates out, and we are left with

$$
\begin{aligned}
\rho(u, v)= & 2 \int_{0}^{\infty} d R \chi_{1}^{*} \chi_{1} N_{2}^{2}\left(\varphi_{3 s}^{*} \varphi_{3 s}+\varphi_{5 p}^{*} \varphi_{5 p}\right. \\
& \left.+2 S_{3 s 5 p} \varphi_{3 s}^{*} \varphi_{5 p}\right)+2 \operatorname{Re}\left(\chi_{1}^{*} \chi_{2}\right) N_{2}\left(\varphi_{3 s}^{*} \varphi_{5 p}\right. \\
& \left.+\varphi_{5 p}^{*} \varphi_{5 p} S_{3 s 5 p}\right)
\end{aligned}
$$

where $S_{3 s 5 p}$ is the overlap between the $3 s$ and $5 p_{z}$ orbital. ${ }^{25}$

In Fig. 1, we have plotted the potentials together with the electron densities at the equilibrium distance of $R_{0}=2.7 \AA$, i.e., Eq. (2) with $\left[\chi_{1}, \chi_{2}\right]$ equal to $\left[\delta\left(R-R_{0}\right), 0\right]$ and $\left[0, \delta\left(R-R_{0}\right)\right]$, respectively.

\section{THE CONTROL SCHEME}

We shall treat $\mathrm{NaI}$ as a one-dimensional system, assuming that the dipole moment is oriented in the direction of the field. The calculation is in three steps. At first we find the vibrational ground state for $\mathrm{NaI}$ in the electronic ground state. Second we use first order perturbation theory to calculate the wave packet in the electronic excited state. Third we calculate the time evolution of the excited wave packet with and without the application of a half-cycle electromagnetic pulse.

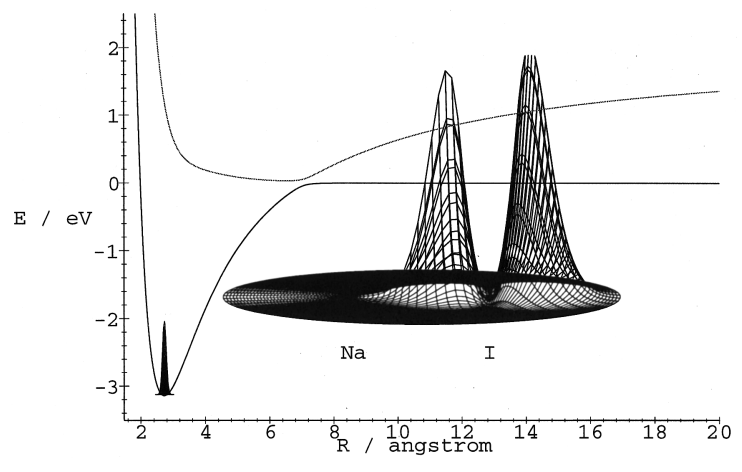

FIG. 2. The vibrational ground state and the corresponding electron density.

\section{A. The vibrational ground state}

We find the vibrational ground state, $\left|\chi^{(0)}\right\rangle$, in the electronic ground state potential by propagating in imaginary time. ${ }^{28}$ The evaluation of the kinetic energy is done using the FFT-method. ${ }^{29}$ The Hamiltonian is

$$
\hat{H}_{1}=-\frac{\hbar^{2}}{2 \mu} \frac{d^{2}}{d R^{2}}+\widetilde{V}_{1}(R)
$$

where $\mu$ is the reduced mass and $\widetilde{V}_{1}$ is the electronic ground state potential in the adiabatic representation. We do the calculation on a 2048 point grid with a spacing of $0.015 \AA$. A fully converged solution can be obtained on a much smaller grid, however, we adapt the size and spacing to the requirements for the calculations to follow. In Fig. 2, we have plotted the vibrational ground state together with the corresponding electron density.

\section{B. The pump pulse}

We electronically excite $\mathrm{NaI}$ from the ground to the excited state using a UV-laser pulse at $\lambda_{1}=328 \mathrm{~nm}$ with Gaussian envelope $a_{1}(t)=\exp \left[-4 \ln 2\left(t / \tau_{1}\right)^{2}\right]$ with a width of $\tau_{1}=25$ fs. The wavelength is taken from the original NaI photodissociation experiment of Zewail and co-workers, ${ }^{9,10}$ and was also used in the calculations published by Engel and Metiu $^{15}$. We calculate the excited wave packet, $\left|\chi^{(1)}\right\rangle$, using first order perturbation theory, assuming the pump pulse to be short enough for the excited wave packet not to interfere with the nonadiabatic coupling. Adopting the rotating wave approximation, we get

$$
\left|\chi^{(1)}\right\rangle=N \int_{-\infty}^{\infty} d t \hat{U}_{2}^{\dagger}(t) \exp \left(-i \omega_{1} t\right) a_{1}(t) \widetilde{D}_{12}\left|\chi^{(0)}\right\rangle,
$$

where $N$ is chosen to normalize $\left|\chi^{(1)}\right\rangle . \omega_{1}$ is defined as $2 \pi c / \lambda_{1}, \widetilde{D}_{12}(R)$ is the electronic transition dipole moment in the adiabatic representation, $\hat{U}_{2}(t)=\exp \left(-i \hat{H}_{2} t / \hbar\right)$ is the propagator and $\hat{H}_{2}$ is the excited state Hamiltonian:

$$
\hat{H}_{2}=-\frac{\hbar^{2}}{2 \mu} \frac{d^{2}}{d R^{2}}+\widetilde{V}_{2}(R),
$$

where $\widetilde{V}_{2}$ is the electronic excited state potential in the adiabatic representation. The time propagation is done using the Lanczos short time propagator ${ }^{30,31}$ and the integration is 


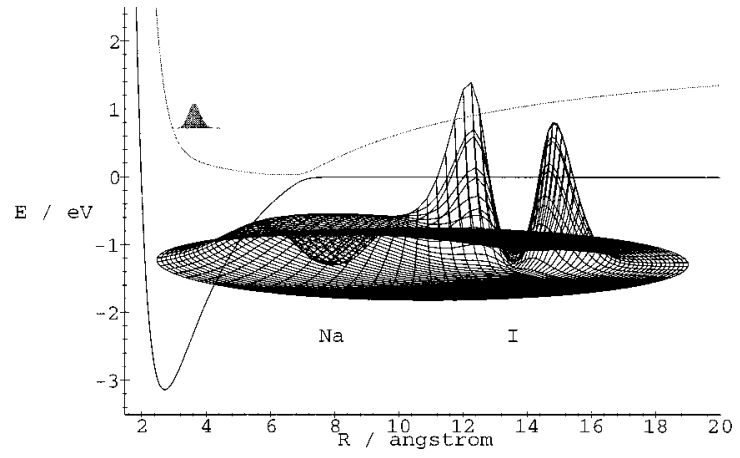

FIG. 3. The excited wave packet and the corresponding electronic density. If we compare with Fig. 2, we see that the excitation corresponds to electron transfer from I to Na.

done using the trapezoidal formula. ${ }^{27}$ In Fig. 3, we have plotted the excited state wave packet together with the corresponding electron density. We see that the excitation corresponds to a partial electron transfer from I to $\mathrm{Na}$, hence, the molecule is now in a covalent state.

\section{The control pulse}

The dynamics of the excited $\mathrm{NaI}$ molecule is governed by motion on two coupled nonadiabatic surfaces. The Hamiltonian in the diabatic representation becomes:

$$
\begin{aligned}
\hat{H}= & -\frac{\hbar^{2}}{2 \mu} \frac{d^{2}}{d R^{2}}+\left[\begin{array}{cc}
V_{1}(R) & V_{12}(R) \\
V_{12}(R) & V_{2}(R)
\end{array}\right]+E(t) \\
& \times\left[\begin{array}{cc}
D_{1}(R) & D_{12}(R) \\
D_{12}(R) & D_{2}(R)
\end{array}\right],
\end{aligned}
$$

where $V_{1}$ and $V_{2}$ are the diabatic potential energy surfaces, $V_{12}$ is the diabatic coupling, $D_{1}$ and $D_{2}$ are the vibrational dipole moments and $D_{12}$ is the electronic transition dipole moment. The subscript 1 denotes the ionic potential and 2 denotes the covalent potential. $E(t)=E_{0} a_{2}(t-\tau) \cos \left[\omega_{2}(t\right.$ $-\tau)]$ is the electric field with envelope $a_{2}(t)=\exp$ $\times\left[-4 \ln 2\left(t / \tau_{2}\right)^{2}\right]$. The initial state is the excited state from the previous section, transformed to the diabatic representation:

$$
\left[\begin{array}{l}
\left|\chi_{1}(0)\right\rangle \\
\left|\chi_{2}(0)\right\rangle
\end{array}\right]=\left[\begin{array}{ll}
W_{11}^{*}(R) & W_{21}^{*}(R) \\
W_{12}^{*}(R) & W_{22}^{*}(R)
\end{array}\right]\left[\begin{array}{c}
0 \\
\left|\chi^{(1)}\right\rangle
\end{array}\right],
$$

where $W_{i j}(R)$ is the diabatic to adiabatic transformation matrix obtained through diagonalization of the diabatic potential energy matrix and where the asterisk denotes complex conjugation.

We do the time propagation in the diabatic representation as the diagonality of the kinetic energy matrix simplifies the evaluation of the Hamiltonian considerably. ${ }^{32}$ We use the Lanczos scheme ${ }^{30,31}$ for each time step. (A typical run $\sim 1 \mathrm{ps}$ takes about $3 \mathrm{~h}$ on a $166 \mathrm{MHz}$ Pentium PC.)

At first we shall see what happens if the control pulse is absent. The molecule will undergo predissociation to form products $\mathrm{Na}$ and I. The calculation so far is actually similar to the one performed by Engel and Metiu ${ }^{16}$ analyzing the results of the famous Zewail experiment on $\mathrm{NaI}$ predissociation. ${ }^{9,10}$
It is, however, instructive to follow more closely what actually happens during one vibration period of $(\mathrm{NaI})^{*}$. In Fig. 4, we have plotted the nuclear configuration together with the electronic density. We see how the electron actually jumps from $\mathrm{Na}$ to I (100-200 fs) and back (700-800 fs) as the molecule vibrates. Further, we see that a small part of the molecule predissociates to form free $\mathrm{Na}$ and I (400-500 fs) each time the wave packet passes through the avoided crossing. It should also be noticed that the electron density as defined in Eq. (2) depends on the nuclear wave packet even when the electronic dynamics is fully adiabatic. For example, at $t=100$ and $800 \mathrm{fs}$ the expectation values of the internuclear distance are similar but the electron density is considerably more delocalized at $t=800 \mathrm{fs}$ due to the highly delocalized nuclear wave packet at that particular time.

From Fig. 4, it is clear that in order to get free $\mathrm{Na}^{+}$and $\mathrm{I}^{-}$we should fire the control pulse in the interval from 200 to $700 \mathrm{fs}$ after the preparation pulse has been fired. We want the control pulse to virtually drag the molecule apart, climbing the Coulomb potential in less than $500 \mathrm{fs}$. Hence it is essential that the breaking of the bond is done in less than a vibration period: Each time the molecule vibrates, yet another part dissociates to form free $\mathrm{Na}$ and I and if we additionally speed up the oscillations with the laser interaction the molecule would tend to follow the diabatic potential, favoring the covalent channel. That is, we do not have an entire vibration period in the molecule to work with and then we cannot tune a laser to be in resonance with the vibration frequency, further, we cannot make an electronic excitation since this would change the electron distribution. It seems clear, that an intense unipolar half-cycle pulse is a good choice for a control pulse. Such a pulse could simply drag the molecule apart in less than one vibration period.

Recently, Bucksbaum et al. ${ }^{33-35}$ have dissociated oriented Rydberg states of $\mathrm{Na}$ and $\mathrm{Cs}$ atoms using intense unipolar half-cycle electromagnetic pulses of a duration of $\tau_{2}$ $=450 \mathrm{fs}$. In this paper we model this pulse, however, with an intensity of $0.5 \mathrm{TW} / \mathrm{cm}^{2}$, which is somewhat higher than that used by Bucksbaum et al. This intensity corresponds to an amplitude of $E_{0}=2 \mathrm{GV} / \mathrm{m}$. The center frequency of the pulse is $\omega_{2}=16.7 \mathrm{~cm}^{-1}$.

In Fig. 5, we see snapshots from a calculation with the time delay $\tau=575$ fs. We see how the wave packet climbs the Coulomb potential and ends with an average energy above $2.08 \mathrm{eV}$ which is the energy required to form ionic products. Further, we see from the electron density that both valence electrons "sit" on I.

Since the energy is quite high, we should address the problem of ionization of the free ionic species. Removing another electron from $\mathrm{Na}^{+}$is out of the question, and removing the electron from $\mathrm{I}^{-}$to form free $\mathrm{Na}^{+}+\mathrm{I}+e^{-}$would require another $3.06 \mathrm{eV}$. The part of the wave packet with energies above this limit is completely negligible, and we conclude that ionization can be neglected.

An animation of the entire calculation illustrating the control scheme can be found on the World Wide Web. ${ }^{36}$

We have made other calculations at different $\tau$ 's and have seen that there is a dependence between $\tau$ and the product outcome. For example, at $\tau=350$ fs the covalent channel 

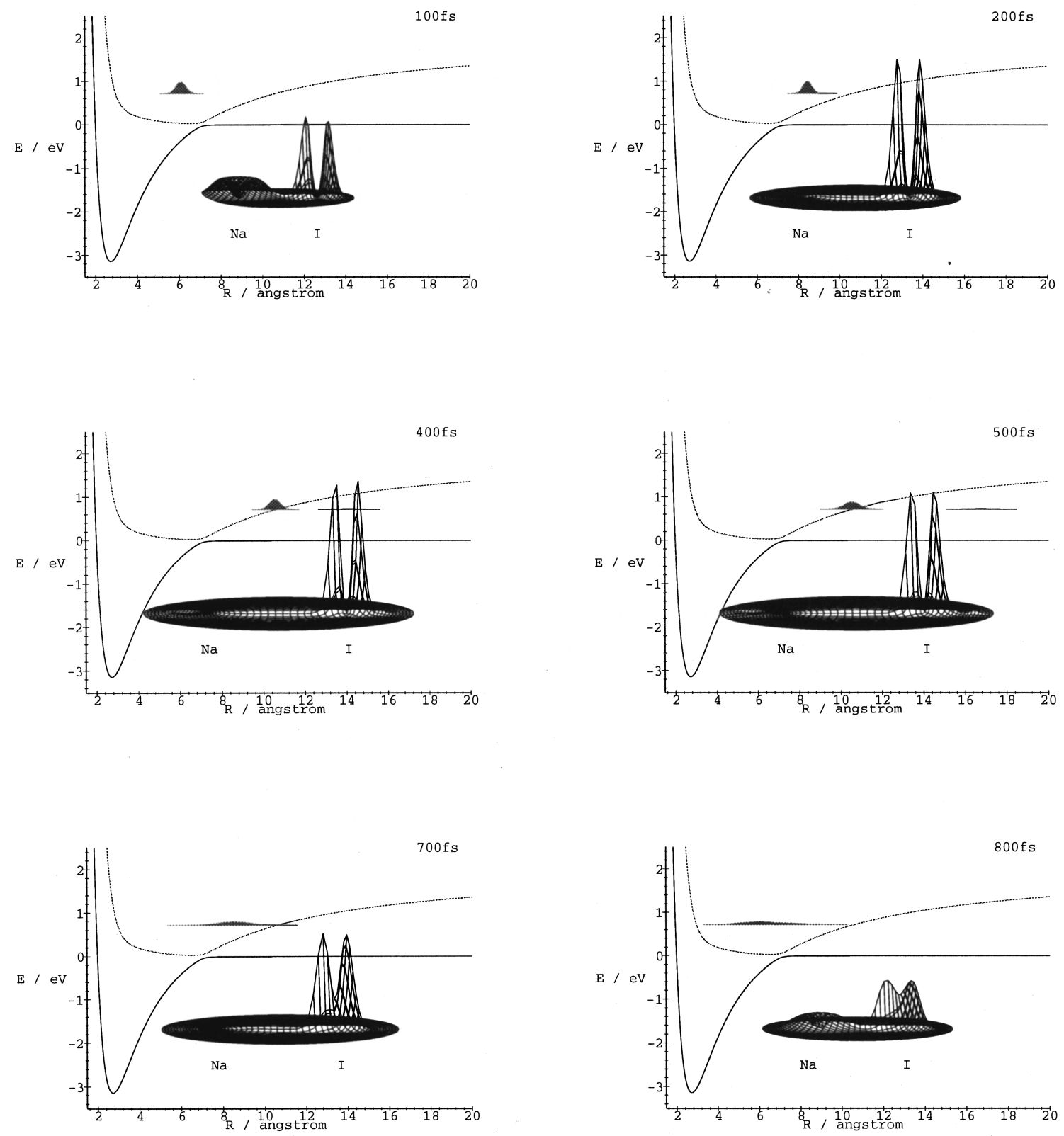

FIG. 4. Snapshots from the time evolution of the excited state with no field present. In the foreground we have plotted the nuclear wave packet and in the background the electronic density. We see that when the wave packet passes the avoided crossing (100-200 fs) one electron is transfered from the $3 s$ orbital of $\mathrm{Na}$ to the $5 \mathrm{p}_{z}$ orbital of I. Further, we see that a small part of the wave packet leaks out to form free Na and I. It is clear from these snapshots that a controlling pulse must be fired between 200 and $700 \mathrm{fs}$ where the electron sits on I.

is favored exclusively. The wave packet simply follows the diabatic potential. This is due to the fact that the electronic transition dipole moment times the field exactly cancels the diabatic coupling potential. This effect has previously been studied in the litterature, ${ }^{37}$ however, not for a realistic system.

\section{DISCUSSION AND CONCLUDING REMARKS}

We have presented a scheme for controlling electron transfer during the breaking of the chemical bond in NaI. The scheme goes as follows: First we create an oscillating electron in the molecule, second we break the bond with a unipolar half-cycle electromagnetic pulse at a given time delay corresponding to the time when the electron "sits" at the right place. The electronic motion is hereby frozen and the electron follows the fragment it was located on just before the bond breakage.

The degree of controllability is very good: Without a control pulse we get $100 \%$ free $\mathrm{Na}$ and I exclusively. With a control pulse fired with the right time delay, we get roughly $95 \%$ free $\mathrm{Na}^{+}$and $\mathrm{I}^{-}$. The $5 \%$ missing predissociates to form free $\mathrm{Na}$ and I before the control pulse is fired.

In this paper, we have demonstrated the principle behind real-time control of electronic motion in a molecule. Rather than simply apply the theory of optimization (see Refs. 4 and $38-41$ ) we have used physical intuition and from that tailored the pulse sequence. Optimization within these ideas would then be the next logical step. 

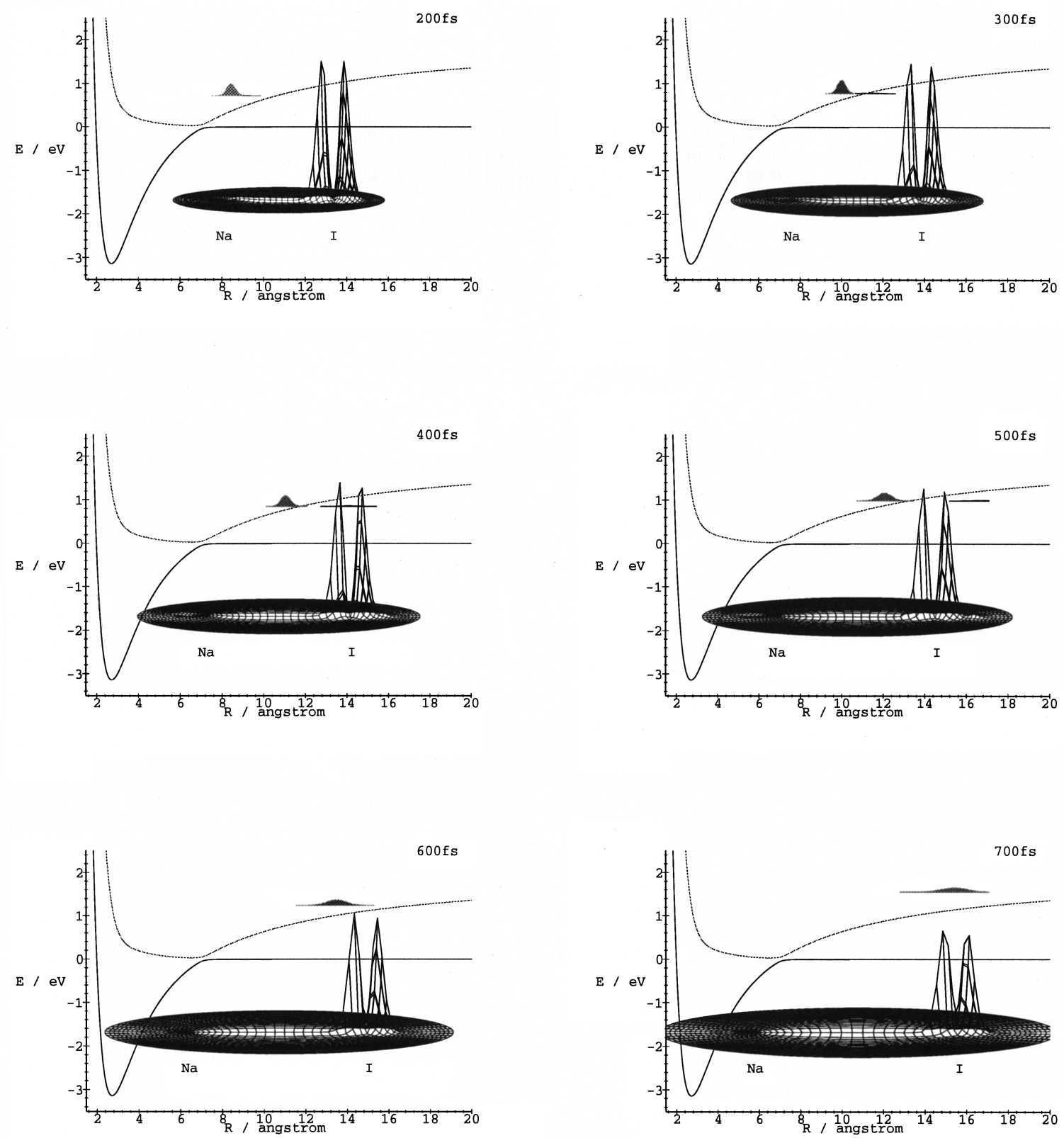

FIG. 5. Snapshots from the time evolution of the excited state with a unipolar half-cycle electromagnetic pulse fired at $\tau=575 \mathrm{fs}$. In the foreground we have plotted the nuclear wave packet and in the background the electronic density. We see a small part of the wave packet escaping as free Na and I due to predissociation, the rest of the wave packet is caught by the field and lifted out above the ionization potential. We see hence that we can control which nucleus the electron is following.

The numbers stated here should, however, be taken with a little precaution. They apply only when we are able to orient the dipole moment of the NaI molecule completely in the direction of the field before firing the control pulse. If we consider a distribution of molecules where all angles between the dipole moment and the field are represented, we would expect the control ratio to be about one-third of the optimal situation. With an aligned angular distribution we would expect the control ratio to be approximately halved. We address this subject in a forthcoming paper. ${ }^{42}$

We have previously examined the scheme and applied it to $\mathrm{HD}^{+}$and found it successful, however, we concluded that even better results could be obtained in systems where the wave packet was able to move entirely through and clear of the avoided crossing, thus making the electron transfer com- plete. This is exactly what we see in NaI: The excited wave packet oscillating in the adiabatic well is entirely clear of the avoided crossing in more than 500 fs which gives us plenty of time to fire the half-cycle pulse and dissociate the molecule into ionic products.

\section{ACKNOWLEDGMENTS}

The authors would like to thank Professor J. T. Hynes for discussions concerning the electronic dipoles of $\mathrm{NaI}$ and Professor Søren R. Keiding for discussions concerning the generation of the half-cycle pulse. This work was supported by the Danish Natural Science Research Council. 
${ }^{1} \mathrm{~J}$. Manz, in Femtochemistry and Femtobiology, edited by V. Sundström (World Scientific, Singapore, 1998).

${ }^{2}$ R. J. Gordon and S. A. Rice, Annu. Rev. Phys. Chem. 48, 601 (1997).

${ }^{3}$ Chemical Reactions and Their Control on the Femtosecond Timescale, Vol. 101 of Advances in Chemical Physics, edited by P. Gaspard and I. Burghardt (Wiley, New York, 1997).

${ }^{4}$ B. Kohler, J. L. Krause, F. Raksi, K. R. Wilson, and V. V. Yakovlev, Acc. Chem. Res. 28, 133 (1995).

${ }^{5}$ M. Grbnager and N. E. Henriksen, Chem. Phys. Lett. 278, 166 (1997).

${ }^{6}$ M. Gronager and N. E. Henriksen, J. Phys. Chem. 102, 4277 (1998).

${ }^{7}$ D. J. Tannor, R. Kosloff, and S. A. Rice, J. Chem. Phys. 85, 5805 (1986).

${ }^{8}$ S. A. Rice, D. J. Tannor, and R. Kosloff, J. Chem. Soc., Faraday Trans. 2 82, 2423 (1986).

${ }^{9}$ T. S. Rose, M. J. Rosker, and A. H. Zewail, J. Chem. Phys. 88, 6672 (1988).

${ }^{10}$ M. J. Rosker, T. S. Rose, and A. H. Zewail, Chem. Phys. Lett. 146, 175 (1988).

${ }^{11}$ J. L. Herek, A. Materny, and A. H. Zewail, Chem. Phys. Lett. 228, 15 (1994).

${ }^{12}$ G. Knopp, M. Schmitt, A. Materny, and W. Kiefer, J. Phys. Chem. 101, 4852 (1997).

${ }^{13}$ C. Jouvet, S. Martrenchard, D. Solgadi, C. Dedonder-Lardeux, M. Mons, G. Grégoire, I. Dimicoli, F. Piuzzi, J. P. Visticot, J. M. Mestdagh, P. D'Oliveira, P. Meynadier, and M. Perdrix, J. Phys. Chem. 101, 2555 (1997).

${ }^{14}$ H. Dietz and V. Engel, Chem. Phys. Lett. 255, 258 (1996).

${ }^{15}$ V. Engel, H. Metiu, R. Almeida, R. A. Marcus, and A. H. Zewail, Chem. Phys. Lett. 152, 1 (1988).

${ }^{16}$ V. Engel and H. Metiu, J. Chem. Phys. 90, 6116 (1989).

${ }^{17}$ G. H. Peslherbe, R. Bianco, J. T. Hynes, and B. M. Ladanyi, J. Chem. Soc., Faraday Trans. 93, 977 (1997).

${ }^{18}$ T. J. Martinez and R. D. Levine, Chem. Phys. Lett. 259, 252 (1996).

${ }^{19}$ T. J. Martinez and R. D. Levine, J. Chem. Phys. 105, 6334 (1996).

${ }^{20}$ C. J. Bardeen, J. Che, K. R. Wilson, V. V. Yakovlev, P. Cong, B. Kohler, J. L. Krause, and M. Messina, J. Phys. Chem. 101, 3815 (1997).
${ }^{21}$ M. B. Faist, B. R. Johnson, and R. D. Levine, Chem. Phys. Lett. 32, 1 (1975).

${ }^{22}$ M. B. Faist and R. D. Levine, J. Chem. Phys. 64, 2953 (1976).

${ }^{23}$ Y. Zeiri and G. G. Balint-Kurti, J. Mol. Spectrosc. 99, 1 (1983).

${ }^{24}$ Y. Sakai, E. Miyoshi, and T. Anno, Can. J. Chem. 70, 309 (1992).

${ }^{25}$ R. S. Mulliken, C. A. Rieke, D. Orloff, and H. Orloff, J. Chem. Phys. 17, 1248 (1949).

${ }^{26}$ E. E. Nikitin and S. Y. Umanskii, Theory of Slow Atomic Collisions (Springer, Berlin, 1984), Vol. 30.

${ }^{27}$ M. A. Abramowitz and I. A. Stegun, Handbook of Mathematical Functions (National Bureau of Standards, Washington, DC., 1964).

${ }^{28}$ R. Kosloff and H. Tal-Ezer, Chem. Phys. Lett. 127, 223 (1986).

${ }^{29}$ R. Kosloff, J. Phys. Chem. 92, 2087 (1988).

${ }^{30}$ C. Leforestier, R. H. Bisseling, C. Cerjan, M. D. Feit, R. Friessner, A. Guldborg, A. Hammerich, G. Jolicard, W. Karrlein, H.-D. Meyer, N. Lipkin, O. Roncero, and R. Kosloff, J. Comput. Phys. 94, 59 (1991).

${ }^{31}$ T. J. Park and J. C. Light, J. Chem. Phys. 85, 5870 (1986).

${ }^{32}$ M. Grønager and N. E. Henriksen, J. Chem. Phys. 104, 3234 (1996).

${ }^{33}$ D. You, R. R. Jones, D. R. Dykaar, and P. H. Bucksbaum, Opt. Lett. 18, 290 (1993).

${ }^{34}$ C. Raman, C. W. S. Conover, C. I. Sukenik, and P. H. Bucksbaum, Phys. Rev. Lett. 76, 2436 (1996).

${ }^{35}$ R. R. Jones, N. E. Tielking, D. You, C. Raman, and P. H. Bucksbaum, Phys. Rev. A 51, R2687 (1995).

${ }^{36} \mathrm{http}: / /$ www.klb.dtu.dk/staff/mpg/movie.mpg.

${ }^{37}$ P. Gross, D. Neuhauser, and H. Rabitz, J. Chem. Phys. 96, 2834 (1992).

${ }^{38}$ Z.-M. Lu and H. Rabitz, J. Phys. Chem. 99, 13731 (1995).

${ }^{39}$ T. T. Nguyen-Dang, C. Chatelas, and D. Tanguay, J. Chem. Phys. 102, 1528 (1995).

${ }^{40}$ H. Shen, J.-P. Dussault, and A. D. Bandrauk, Chem. Phys. Lett. 221, 498 (1994).

${ }^{41}$ B. Amstrup, G. J. Tóth, G. Szabó, H. Rabitz, and A. Lőrincz, J. Phys. Chem. 99, 5206 (1995).

${ }^{42}$ M. Grønager and N. E. Henriksen (unpublished). 